TRANSACTIONS OF THE

AMERICAN MATHEMATICAL SOCIETY

Volume 188, Issue 2, 1974

\title{
UNIFORMLY DISTRIBUTED SEQUENCES IN LOCALLY COMPACT GROUPS. II
}

BY

\section{LEONORA BENZINGER}

ABSTRACT. We consider the following question. When is there a compactification $G_{0}$ of a locally compact group $G$ (recall that a compact group $G_{0}$ is a compactification of $G$ if there is a continuous homomorphism $\phi: G \rightarrow G_{0}$ so that $\phi(G)$ is dense in $G$ ) with conxinuous homomorphism $\phi: G-G_{0}$ with the property that $\left\{g_{v}\right\}$ is uniformly distributed in $G$ if and only if $\left\{\phi\left(g_{v}\right)\right\}$ is uniformly distributed in $G_{0}$ ? Such a compactification $G_{0}$ is called a $D$-compactification of $G$. We obtain a solution to this problem and thereby generalize to locally compact groups some results of Berg, Rajagopalan, and Rubel concerning $D$-compactifications of locally compact abelian groups.

Introduction. In the first section we discuss compactifications of locally compact groups in terms of the maximal ideal spaces of associated function algebras and we introduce the notion of almost closed sets of finite dimensional unitary representations. In the second section we develop some of the standard ideas concerning compactifications to obtain some preliminary lemmas relating almost closed subsets of finite dimensional unitary representations and compactifications of locally compact groups. In the third section we use the machinery developed in the first two sections to obtain results concerning the existence and uniqueness of $D$-compactifications for locally compact groups which admit uniformly distributed sequences. A generalization of the key lemma of [1] plays a crucial role in the proof of these results. We shall use the definitions and notation which appear in [1].

1. Preliminary definitions and results. We now present some ideas which are useful in discussing compactifications of locally compact groups.

We observe that for each representation $\rho$ in $R(G)[1, \S 1]$ the conjugate $\bar{\rho}$ defined by $\bar{\rho}(g)=\overline{\rho(g)}$ for each $g$ in $G$ is also in $R(G)$.

Let $S \subset R(G)$. By $\bar{S}$ we mean $\{\bar{\rho} \mid \rho \in S\}$. Let $A(S)$ denote the algebra generated by the m.a.i. functions $\left[1, \S_{1}\right]$ from representations in $S \cup \bar{S}$. Let $B A(S)$ denote the closure of $A(S)$ with respect to the uniform norm. We shall call $B A(S)$ the Banach algebra generated by $S$.

Received by the editors February 13, 1973.

AMS (MOS) subject classifications (1970). Primary 22D05; Secondary $10 \mathrm{~K} 99$.

Key words and phrases. Locally compact group, uniform distribution, compactification, $D$-compactification.

Copyright $\odot$ 1974, Americen Mathereatical Socibty 
Let $f$ be any complex valued function defined on a topological group $G_{0}$. The function $f_{b}$ is defined by $f_{b}(x)=f(x b)$ for a fixed $b$ in $G_{0}$ and is called the right translate of $f$ by $b$. The function $f_{b}$ is defined by $f_{b}(x)=f(a x b)$ for all $x$ in $G_{0}$ and is called the mixed translate of $f$ by $a$ and $b$. The translates of the finite dimensional unitary representations of a locally compact group $G$ are defined in the obvious way. An algebra $A$ of functions on $G_{0}$ is translation invariant if for each $f \in A$ and each $a, b \in G_{0}, f_{b} \in A$.

Remark. Let $\rho_{i j} \in A(S)$ be a m.a.i. function of some representation $\rho$ in $S U$ $\bar{S}$ and let $a, b \in G$. Then the function $\left({ }_{a} \rho_{b}\right)_{i j}$ lies in the subspace generated by the m.a.i. functions of $\rho$ and $\left({ }_{a} \rho_{b}\right)_{i j} \in A(S)$. Indeed, for each $x \in G$

$$
\left({ }_{a} \rho_{b}\right)_{i j}(x)=\sum_{s, k=1}^{m} \rho_{i k}(a) \rho_{s j}(b) \rho_{k s}(x) .
$$

The Banach algebra generated by $S, B A(S)$, is a commutative translation invariant algebra with an identity and conjugation as an involution. Indeed, from the preceding remark it follows that $A(S)$ is translation invariant. We then use the fact that $A(S)$ is dense in $B A(S)$ to show that $B A(S)$ is translation invariant.

A continuous complex valued function $f$ on $G_{0}$ is almost periodic if the closure in the uniform norm of $\left\{f_{a} \mid a \in G_{0}\right\}$ is compact. We denote the set of all almost periodic functions on $G_{0}$ by $A P\left(G_{0}\right)$.

From Loomis [5, Theorem, p. 88] we see that $B A(S)$ is isomorphically isometric to $C\left(M_{S}\right)$, the continuous functions on the compact Hausdorff space $M_{S}$ of its maximal ideals. The existence of a continuous homomorphism $a$ taking $G$ into $M_{S}$ so that $\alpha(G)$ is dense in $M_{S}$ is clear. It is not obvious, however, that $M_{S}$ is actually a compactification of $G$. We have a situation similar to that in Loomis $[5, \S 41]$ which is a discussion of the Bohr compactification of a topological group.

(1.1) Lemma. Let $G$ be a locally compact group and let $S \subset R(G)$. Form $B A(S)$, the Banach algebra generated by $S$. The maximal ideal space $M_{S}$ of $B A(S)$ is a compactification of $G$ and is unique up to isometric isomorphism.

Proof. We show first that each m.a.i. function from a representation in $S$ is almost periodic. Let $\rho \in S$ be of dimension $m$. Define $T: \rho_{a} \rightarrow\left[\left(\rho_{a}\right)_{i j}\right]$. The mapping is 1 to 1 so that $T^{-1}$ exists. Then

$$
\left\|T\left(\rho_{a}\right)-T\left(\rho_{b}\right)\right\|=\left\|T^{-1}\left(\left[\left(\rho_{a}\right)_{i j}\right]\right)-T^{-1}\left(\left[\left(\rho_{b}\right)_{i j}\right]\right)\right\| \text {. }
$$

From this equality it follows that $T$ and $T^{-1}$ are continuous. In addition $T^{-1}$ is linear. Let $U=\left\{\left[\left(\rho_{a}\right)_{i j}\right] \mid a \in G\right\}$. There exists a unique continuous extension of $T^{-1}$ to $\bar{U}$. Because $\rho$ is unitary, $\bar{U}$ is a bounded subset of the set $U(m)$ of $m \times m$ unitary matrices. Hence $\bar{U}$ is compact. The set $T^{-1}(\bar{U})$ is compact and therefore closed. Let $V=\left\{\rho_{a} \mid a \in G\right\}$. Since $\bar{V} \subset T^{-1}(\bar{U}), \bar{V}$ is compact. 
We define a projection mapping $P_{i j}: \rho_{a} \rightarrow\left(\rho_{a}\right)_{i j}$. Since the mapping $P_{i j}$ is linear and continuous, there exists a unique continuous extension to $\bar{V}$. The set $P_{i j}(\bar{V})$ is compact and hence closed. The set $\bar{U}$ is a closed subset of $P_{i j}(\bar{V})$ and therefore is compact. Thus $\rho_{i j}$ is almost periodic. By similar reasoning, $\bar{\rho}_{i j}$ is also almost periodic. Therefore each generator of the algebra $A(S)$ lies in $A P(G)$. Since $A P(G)$ is itself closed with respect to the uniform norm and contains $A(S)$, $A P(G)$ contains $B A(S)$.

By using reasoning very similar to Loomis $[5, \S 41]$ we see that the maximal ideal space $M$ of any translation invariant closed subalgebra $A$ of $A P(G)$ is a compactification of $G$ and is unique up to isometric isomorphism. We complete the proof by observing that $B A(S)$ is a translation invariant closed subalgebra of $A P(G)$.

(1.2) Notation. Let $S \subset R(G)$. We shall denote the compactification of $G$ obtained as the maximal ideal space of $B A(S)$ by $G_{S}$. We note that it is possible to have two distinct subsets $S_{0}$ and $S_{1}$ of $R(G)$ such that $B A\left(S_{0}\right)=B A\left(S_{1}\right)$. Then, of course, $G_{S_{0}}=G_{S_{1}}$.

The construction given yields all compactifications of $G$. That is, each compactification of $G$ can be thought of as the maximal ideal space of some translation invariant Banach algebra with an involution and an identity which is generated by the m.a.i. functions of some subset of $R(G)$.

We now give some examples of compactifications of $G$. The Bobr compactification of $G$ is the maximal ideal space of $A P(G)$. It may be considered as the maximal ideal space of $B A(R(G))$ and is isomorphically isometric to $G_{R(G)}$. The trivial compactification $\{0\}$ may be obtained as the maximal ideal space of $B A(\{\rho\})$ where $\rho$ is the trivial representation of $G$; that is, $\rho(G)=1$. The next example is a generalization to locally compact groups of the notion of periodic compactification found in [2]. Form $B A(P)$, the Banach algebra generated by $P$, the periodic irreducible unitary representations of $G$ [1]. The maximal ideal space $G_{P}$ of $B A(P)$ is called the periodic compactification of $G$.

If $G$ is abelian, we can obtain $G_{P}$ as the compact dual of the discrete subgroup of $G^{\wedge}$ generated by the periodic characters of $G$, see [2, p. 436].

We now introduce some definitions.

(1.3) Definition. Let $S$ be a subset of finite dimensional (not necessarily irreducible) unitary representations of a locally compact group $G$. If the product of any two m.a.i. functions from representations in $S$ is a finite linear combination of m.a.i. functions from representations in $S$ we say that the m.a.i. functions from representations in $S$ are almost closed with respect to products. If the conjugate of each m.a.i. function from a representation in $S$ is a finite linear combination of m.a.i. functions from representations in $S$ we say that the m.a.i. functions of representations in $S$ are almost closed with respect to conjugation. 
(1.4) Definition. A set $S$ of finite dimensional unitary representations of a locally compact group $G$ is an AC (almost closed) set if the m.a.i. functions of representations in $S$ are almost closed with respect to products and conjugation.

Remark. For a slightly different approach, it is possible to define a type of multiplication $\times$ on $R(G)$ and then for each subset $S \subset R(G)$ consider the smallest subset of $R(G)$ containing $S$ which is closed with respect to $\times$ and conjugation. For example, for compact $G$ see Hewitt and Ross [4] and in particular [4, Definition 27.35].

We now state our main result.

Theorem. Let $G$ be a locally compact group which admits a u.d. sequence and let $P$ be an $A C$ subset of $R(G)$. Then a sequence $\left\{x_{v}\right\}$ in $G$ is $u_{0} d$. if and only if $\left\{\phi\left(x_{v}\right)\right\}$ the image of $\left\{x_{v}\right\}$ under the natural bomomorphism from $G$ into $G_{P}$ is u.d. in $G_{P}$. In addition, $G_{P}$ is the only compactification of $G$ which is a D-compactification. If $P$ is not an $A C$ subset of $R(G)$ then there is no D-compactification.

2. AC subsets of finite dimensional unitary representations. The definitions and lemmas which follow yield three different characterizations of $A C$ subsets of finite dimensional unitary representations of a locally compact group $G$.

(2.1) Lemma. Let $S$ be an $A C$ subset of the finite dimensional unitary representations of $G$. Then eacb $f \in A(S)$ can be expressed as a finite linear combination of m.a.i. functions from representations in $S$.

Proof. It suffices to show that products of the form $f_{1} f_{2} \cdots f_{k}$ where each $f_{i}, 1 \leq i \leq k$, is a m.a.i. function from some $\rho$ in $S$ or is a conjugate of a m.a.i. function from some $\rho$ in $S$ are finite linear combinations of m.a.i. functions from representations in $S$. Any product of m.a.i. functions from representations in $S$ is a linear combination of m.a.i. functions from representations in $S$. This follows by an induction argument on the number $n$ of nontrivial factors which appear in a product as m.a.i. functions from representations in $S$. By similar reasoning, any product of conjugates of m.a.i. functions from representations in $S$ is also a linear combination of m.a.i. functions from representations in $S$. We write $f_{1} f_{2} \cdots$ $f_{k}$ as $f g$ where $f$ is a product of m.a.i. functions from representations in $S$ and $g$ is a product of conjugates of m.a.i. functions which are not themselves m.a.i. functions from representations in $S$. From the fact that the m.a.i. functions from representations in $S$ are almost closed with respect to products it follows that $\mathrm{fg}$ is a finite linear combination of m.a.i. functions from representations in $S$.

Remark. As a consequence of Lemma (2.1), an AC subset $S$ of finite dimensional unitary representations of $G$ all of whose nontrivial m.a.i. functions are orthogonal to 1 contains the trivial representation. Indeed, let $\rho$ be a member of 
$S$ of dimension $m$ and suppose that $\rho=\left[\rho_{i j}\right]$. The function $\Sigma_{k=1}^{m} \rho_{i j} \overline{\rho_{i k}} \in A(S)$. Since $\rho$ is unitary, $\Sigma_{k=1}^{m} \rho_{i k} \overline{\rho_{i k}}=1$. The function 1 by Lemma $(2.1)$ is a finite linear combination of m.a.i. functions from representations in $S$. This is only possible if $1 \in S$. As a special case, we observe that an AC subset of $R(G)$ contains the trivial representation.

(2.2) Notation. Let $S \subset R(G)$. Then $T(S)$ is the set of all finite tensor products of members of $S$. It is well known that the tensor product of two finite dimensional irreducible unitary representations is a finite dimensional representation, for example, see [6, p. 436]. The tensor product representation may or may not be reducible.

(2.3) Definition. Let $S \subset R(G)$ and form $T(S)$. A representation $\rho$ of $R(G)$ is said to be obtained from $T(S)$ if there exists a $\sigma \in T(S)$ with a reduction in terms of irreducible unitary representations at least one of which is equivalent to $\rho$.

The lemma which follows is our first characterization of AC subsets of $R(G)$.

(2.4) Lemma. Let $S \subset R(G)$. The set $S$ is an $A C$ subset of $R(G)$ if and only if the finite dimensional irreducible unitary representations of $G$ which can be obtained from $T(S \cup \bar{S})$ lie in $S$.

Proof. Suppose that $S$ is an $A C$ subset of $R(G)$ and that there exists a representation $\rho$ obtained from $T(S \cup \bar{S})$ which does not lie in $S$. There exists a $\sigma \in T(S \cup \bar{S})$ and $\sigma_{1}, \sigma_{2}, \cdots, \sigma_{k}$ each of which is in $R(G)$ and a constant unitary matrix $c$ so that

$$
\sigma=c\left[\begin{array}{llll}
\sigma_{1} & & & \\
& \sigma_{2} & \\
& & \ddots & \\
& & \sigma_{k}
\end{array}\right] c^{-1}
$$

In addition, $\rho$ is equivalent to $\sigma_{i}$ for some $i, 1 \leq i \leq k$. Each m.a.i. function of $\sigma_{i}$ lies in $A(T(S \cup \bar{S}))$. Therefore any m.a.i. function $f$ of $\rho$ is a finite linear combination of members of $A(T(S \cup \bar{S}))$ and hence lies in $A(T(S \cup \bar{S}))$. Since $A(S)=A(T(S \cup \bar{S}))$ and $S$ is an $A C$ subset of $R(G), f$ is a finite linear combination of m.a.i. functions from representations in $S$ by Lemma (2.1). This is not possible because any m.a.i. function of a member of $R(G)$ not in $S$ is orthogonal to all m.a.i. functions of representations in $S$.

Next, let us assume that the irreducible unitary representations of $G$ which can be obtained from $T(S \cup \bar{S})$ lie in $S$. Let $f$ and $g$ be m.a.i. functions of $\rho$ and $\sigma$ which are (not necessarily distinct) representations in $S$. Then $f g$ is a m.a.i. function of $\rho \otimes \sigma$ in $T(S \cup \bar{S})$. There exist $\sigma_{1}, \sigma_{2}, \cdots, \sigma_{k}$ each of which is in $S$ and a constant unitary matrix $c$ such that 


$$
\rho \otimes \sigma=c\left[\begin{array}{llll}
\sigma_{1} & & & \\
& & & \\
& \sigma_{2} & \\
& & \ddots & \\
& & \sigma_{k}
\end{array}\right] c^{-1}
$$

Therefore $f g$ is a finite linear combination of m.a.i. functions from representations in $S$. Thus the m.a.i. functions from representations in $S$ are almost closed with respect to products. For each $\rho \in S, \bar{\rho} \in T(S \cup \bar{S}) \cap R(G)$. Since $\bar{\rho}$ is a member of $R(G)$ which is obtained from $T(S \cup \bar{S}), \bar{\rho} \in S$. It follows that the m.a.i. functions from representations in $S$ are almost closed with respect to conjugation. Therefore $S$ is an $A C$ subset of $R(G)$.

Remark. Lemma (2.4) gives a characterization of AC subsets of $R(G)$ in terms of tensor products. The tensor product formulation is a more natural generalization of the analogue to AC subsets of $R(G)$ in the case that $G$ is abelian. It is easily seen that if $G$ is abelian, the $A C$ subsets of the character group $G^{\wedge}$ are exactly the subgroups of $G^{\hat{}}$. If $G$ is abelian Lemma (2.4) reads as follows:

Let $S \subset G^{\wedge}$. The set $S$ is an $A C$ subset of $G^{\wedge}$ if and only if the characters in $S$ are closed with respect to products and inverses; that is, if and only if $S$ is a subgroup of $G^{\wedge}$.

For purposes of proof, $A C$ subsets of $R(G)$ are very easy to work with directly in terms of their definition and we shall use the definition rather than the tensor product characterization in the lemmas which follow.

(2.5) Lemma. Let $S$ be a subset of $R(G)$. The Banach algebra $B A(S)$ contains only those m.a.i. functions from representations in $R(G)$ which lie in $S$ if and on$l y$ if $S$ is an $A C$ subset of $R(G)$.

Proof. Assume that $S$ is an AC subset of $R(G)$. Suppose $g$ is a m.a.i. function from some representation $\rho$ in $R(G)-S$ and that $g \in B A(S)$. Since $A(S)$ is dense in $B A(S)$, given any $\epsilon>0$ there exists $f_{0} \epsilon A(S)$ such that $\left|g(x)-f_{0}(x)\right|<\epsilon$ for all $x$ in $G$. By Lemma (2.1) there exist complex numbers $a_{0}, a_{1}, \cdots, a_{m}$ and nontrivial m.a.i. functions $g_{1}, g_{2}, \cdots, g_{m}$ from representations in $S$ such that $f_{0}=a_{0}+\sum_{i=1}^{m} a_{i} g_{i}$. Let us consider $g$ and each $g_{i}$ as functions on the compactification $G_{S}$ and let $\mu$ be normalized Haar measure on $G_{s} \cdot$ Set

$$
g(x)=f(x)+a_{0}+\sum_{i=1}^{m} a_{i} g_{i}
$$

The function $f$ is continuous and $\sup |f(x)| \leq \epsilon$. If $r$ is the dimension of the representation $\rho \in R(G)$ which admits $g$ as a m.a.i. function, then it is known that 
$\int_{G_{S}} g \bar{g} d \mu=1 / r$. Select $\epsilon$ so that it is less than $1 / \sqrt{r}$. We have

$$
1 / r=\left|\int_{G_{S}} g(x) \bar{f}(x) d \mu\right| \leq\|f\|_{2}\|g\|_{2} \leq \epsilon / \sqrt{r} .
$$

This is not possible by the choice of $\epsilon$.

Next, assume that $B A(S)$ contains only those m.a.i. functions from representations in $R(G)$ which lie in $S$. It follows that $A(S)$ contains only those m.a.i. functions from representations in $R(G)$ which lie in $S$. The m.a.i. functions from representations in $S$ are almost closed with respect to products. Indeed, let $g_{1}$ and $g_{2}$ be m.a.i. functions from representations in $S$ and suppose that $g_{1} g_{2}$ is not a linear combination of m.a.i. functions from representations in $S$. Since $R(G)$ is itself an AC set, by Lemma (2.1), $g_{1} g_{2}=\sum_{i=1}^{n} \alpha_{i} f_{i}$ where each $f_{i}$ is a m.a.i. function from a representation in $R(G)$. At least one such function say $f_{i_{0}}$ does not come from a representation in $S$. But then $A(S)$ contains $f_{i_{0}}$ which is a contradiction. By similar reasoning it follows that the m.a.i. functions from representations in $S$ are almost closed with respect to conjugation. Hence $S$ is an AC subset of $R(G)$.

Remark. For each $\rho \in P$ it follows that $\bar{\rho} \in P$. Therefore by Lemma (2.5), the Banach algebra generated by the periodic representations of $R(G), B A(P)$, contains only those m.a.i. functions from representations in $R(G)$ which lie in $P$ if and only if the m.a.i. functions from representations in $P$ are almost closed with respect to products. If $G$ is abelian, then $B A(P)$ contains only periodic characters if and only if $P$ is a group.

Let $G$ be a locally compact group and let $S \subset R(G)$. We form $G_{S}$, the compactification of $G$ with respect to $S$. It is sometimes convenient to consider $R\left(G_{S}\right)$ as a subset of $R(G)$. We note that $S$ is then a subset of $R\left(G_{S}\right)$. In this setting we have the third and last characterization of AC subsets of $R(G)$.

(2.6) Lemma. Let $S \subset R(G)$ and let $G_{S}$ be the compactification of $G$ with respect to $S$. The set $S$ is an $A C$ subset of $R(G)$ if and only if $S=R\left(G_{S}\right)$.

Proof. We assume that $S$ is an AC subset of $R(G)$. It is clear from the discussion on compactifications that $B A(S)$ is topologically isomorphic to $C\left(G_{S}\right)$. We see by the Peter-Weyl theorem that $C\left(G_{S}\right)$ is topologically isomorphic to $B A\left(R\left(G_{S}\right)\right)$. Thus $B A(S)$ is topologically isomorphic to $B A\left(R\left(G_{S}\right)\right)$. By Lemma (2.5) the subset of $R(G)$ all of whose m.a.i. functions lie in $B A(S)$ is exactly $S$. The set $R\left(G_{S}\right)$ consists of all equivalence classes of irreducible unitary representations of $G_{S}$ all of whose m.a.i. functions lie in $B A\left(R\left(G_{S}\right)\right)$. Therefore $S=R\left(G_{S}\right)$.

If $S=R\left(G_{S}\right)$, then $B A(S)=B A\left(R\left(G_{S}\right)\right)$. The Banach algebra $B A\left(R\left(G_{S}\right)\right)$ contains only those m.a.i. functions from representations in $R(G)$ which are m.a.i. 
functions from representations in $R\left(G_{S}\right)$. Therefore $B A(S)$ contains only those m.a.i. functions from representations in $R(G)$ which are m.a.i. functions from representations in $S$. By Lemma (2.5), $S$ is an AC subset of $R(G)$.

3. D-compactifications of locally compact groups which admit uniformly distributed sequences. In this section we present two preliminary lemmas and a theorem concerning the existence and uniqueness of $D$-compactifications of locally compact groups which admit u.d. sequences (these are exactly the $K$-separable [1] locally compact groups).

We recall that for each $S \subset R(G)$ the set $T(S)$ is all finite tensor products of representations in $S$. For the notation $\left\langle\rho,\left\{x_{v}\right\}\right\rangle$, see $[1, \S 1]$.

(3.1) Lemma. Let $G$ be a locally compact group, let $Q$ be an $A C$ subset of $R(G)$, and let $\sigma \in R(G)-Q$. Suppose that there exists a sequence $\left\{x_{v}\right\}$ in $G$ sucb that $\left\langle\rho,\left\{x_{v}\right\}\right\rangle=0$ for all nontrivial $\rho \in Q$. Form $B A(Q \cup\{\sigma\})$, the Banacb algebra generated by $Q \cup\{\sigma\}$ and let $M=G_{Q \cup\{\sigma\}}$, the compactification of $G$ witb respect to $Q \cup\{\sigma\}$. Let $S$ be that subset of $R(G)$ whose representations we identify witb those of $R(M)$. Then there is a sequence $\left\{b_{v}\right\}$ in $G$ sucb tbat for eacb nontrivial m.a.i. function $g$ from a representation in $S,\left\langle g,\left\{b_{\nu}\right\}\right\rangle$ exists and equals 0.

Proof. We start by proving that there exists a sequence $\left\{y_{v}\right\}$ in $G$ such that for each nontrivial m.a.i. function $f$ from a representation $\rho \in T(Q \cup\{\sigma\} \cup\{\bar{\sigma}\})$ there is a $y_{v} \in\left\{y_{v}\right\}$ such that $f\left(y_{v}\right) \neq 1$. For each m.a.i. function $f$ from a nontrivial $\rho$ in $Q$ there is an $x_{v}$ in $\left\{x_{v}\right\}$ such that $f\left(x_{v}\right) \neq 1$. Let $a$ be the continuous homomorphism mapping $G$ into its compactification $G_{Q}$. By Lemma (2.6), $R\left(G_{Q}\right)=Q$. Then $\left\langle\rho,\left\{x_{v}\right\}\right\rangle=0$ for all nontrivial $\rho \in Q$ implies that $\left\langle\rho,\left\{a\left(x_{v}\right)\right\}\right\rangle=0$ for all nontrivial $\rho \in R\left(G_{Q}\right)$. Thus $\left\{a\left(x_{v}\right)\right\}$ satisfies the Weyl criterion for compact groups $[1, \S 1]$ and $\left\{a\left(x_{\nu}\right)\right\}$ is u.d. in $G_{Q}$. On a compact group a u.d. sequence is dense. Therefore $\overline{\left\{a\left(x_{v}\right)\right\}}=G_{Q}$. For each pair of continuous complex valued functions $\hat{f}, g^{\wedge}$ on $G_{Q}$ there is an $x_{v}$ such that $\hat{f^{\wedge}}\left(a\left(x_{v}\right)\right) \neq g^{\wedge}\left(\alpha\left(x_{v}\right)\right)$. If $f$ and $g$ are the functions in $B A(Q)$ corresponding to $f^{\wedge}$ and $g^{\wedge}$ respectively, then $f\left(x_{v}\right) \neq$ $g\left(x_{v}\right)$.

We now construct a sequence which separates the nontrivial m.a.i. functions from representations in $T(Q \cup\{\sigma\} \cup\{\bar{\sigma}\})$ from 1 . Let

$$
\sigma=\left[\begin{array}{llll}
\sigma_{1} & \sigma_{2} & \cdots & \sigma_{m} \\
\sigma_{m+1} & \sigma_{m+2} & \cdots & \sigma_{2 m} \\
\vdots & \vdots & \cdots & \vdots \\
\sigma_{m}^{2}-m+1 & \sigma_{m}^{2}-m+2 & \cdots & \sigma_{m}^{2}
\end{array}\right]
$$


A m.a.i. function from a representation in $T(Q \cup\{\sigma\} \cup\{\bar{\sigma}\})$ can be written as $g \Pi_{i=1}^{m 2} \sigma_{i}^{n_{i}} \bar{\sigma}_{i}^{k_{i}}$ where $n_{i}$ and $k_{i}$ are nonnegative integers for each $i$ and $g \in B A(Q)$ is a product of nonnegative powers of m.a.i. functions from representations in $Q$. For all $g \in B A(Q)$ except at most one, there exists $x_{v}$ in $\left\{x_{v}\right\}$ such that

$$
g\left(x_{v}\right) \prod_{i=1}^{m^{2}} \sigma_{i}^{n_{i}}\left(x_{v}\right) \bar{\sigma}_{i}^{k}\left(x_{v}\right) \neq 1 \text {. }
$$

Indeed, suppose that $g_{0}$ and $g_{1} \in B A(Q)$ and

$$
g_{0}\left(x_{v}\right) \prod_{i=1}^{m^{2}} \sigma_{i}^{n_{i}}\left(x_{v}\right) \bar{\sigma}_{i}^{k} i\left(x_{v}\right)=g_{1}\left(x_{v}\right) \prod_{i=1}^{m^{2}} \sigma_{i}^{n_{i}}\left(x_{v}\right) \bar{\sigma}_{i}^{k}\left(x_{v}\right)=1
$$

for all $x_{v} \in\left\{x_{v}\right\}$. It follows that $g_{0}\left(x_{v}\right)=g_{1}\left(x_{v}\right)$ for all $x_{v}$ in $\left\{x_{v}\right\}$ and hence $\boldsymbol{g}_{0}=\boldsymbol{g}_{1}$.

By adjoining one more element $w$ of $G$ to $\left\{x_{v}\right\}$ we obtain a set separating each m.a.i. function of the form $g \Pi_{i=1}^{m^{2}} \sigma_{i}^{n_{i}} \bar{\sigma}_{i}^{k_{i}}$ from 1. As $n_{i}$ and $k_{i}$ run through all nonnegative integers we obtain all possible m.a.i. functions from representations in $T(Q \cup\{\sigma\} \cup\{\bar{\sigma}\})$. For each $2 m^{2}$-tuple of nonnegative integers we adjoin at most a single element $w\left(n_{1}, n_{2}, \cdots, n_{m_{2}}, k_{1}, k_{2}, \cdots, k_{m_{2}}\right)$ to $\left\{x_{v}\right\}$ so as to obtain a countable set $\left\{y_{v}\right\}$ in $G$ which separates all nontrivial m.a.i. functions from representations in $T(Q \cup\{\sigma\} \cup\{\bar{\sigma}\})$ from 1. Let $H$ be the countable subgroup generated by $\left\{y_{v}\right\}$. By $\left[1\right.$, Lemma 3.3] there exists a sequence $\left\{b_{v}\right\}$ in $H$ such that $\left\langle f,\left\{b_{v}\right\}\right\rangle=0$ for each nontrivial m.a.i. function $/$ from a representation in $T(Q \cup\{\sigma\} \cup\{\bar{\sigma}\})$.

We observe that all nontrivial m.a.i. functions in $T(Q \cup\{\sigma\} \cup\{\bar{\sigma}\})$ are orthogonal to 1. It is a straightforward matter to verify that $T(Q \cup\{\sigma\} \cup\{\bar{\sigma}\})$ is an $A C$ subset of the finite dimensional unitary representations of $G$. By Lemma (2.1) each function in $A(T(Q \cup\{\sigma\} \cup\{\bar{\sigma}\}))$ is a finite linear combination of m.a.i. functions from representations in $T(Q \cup\{\sigma\} \cup\{\bar{\sigma}\})$. Since $A(Q \cup\{\sigma\})=A(T(Q \cup\{\sigma\} \cup\{\bar{\sigma}\}))$, for each $f \epsilon$ $A(Q \cup\{\sigma\})$ which is orthogonal to 1 , there exist scalars $a_{1}, a_{2}, \cdots, a_{s}$ and nontrivial m.a.i. functions $f_{1}, f_{2}, \cdots, f_{s}$ from representations in $T(Q \cup\{\sigma\} \cup\{\bar{\sigma}\})$ such that $f=\Sigma_{i=1}^{s} a_{i} f_{i}$. Then, $\left\langle f,\left\{b_{v}\right\}\right\rangle=\Sigma_{i=1}^{s} a_{i}\left\langle f_{i},\left\{b_{v}\right\}\right\rangle=0$.

It follows from the fact that $A(Q \cup\{\sigma\})$ is dense in $B A(Q \cup\{\sigma\})$ that $\left\langle g,\left\{b_{\nu}\right\}\right\rangle=0$ for each $g \in B A(Q \cup\{\sigma\})$ which is orthogonal to 1 . We complete the proof by observing that each nontrivial m.a.i. function from a representation in $S$ is an element of $B A(Q \cup\{\sigma\})$ which is orthogonal to 1.

Remark. It may be of interest to compare Lemma (3.1) with the abelian analogue [2, Lemma 2.2, p. 440].

(3.2) Lemma. Let $H$ be a compact group which admits a $u_{0} d$ sequence $\left\{y_{v}\right\}$. 
Let $\sigma$ be a nontrivial member of $R(H)$. Then there exists a sequence $\left\{x_{v}\right\}$ in $H$ such that $\left\langle\rho,\left\{x_{v}\right\}\right\rangle=0$ for all nontrivial $\rho \in R(H)$ except $\sigma$ and $\bar{\sigma}$.

Proof. Let $\sigma=\left[\sigma_{i j}\right]$ and set $\psi(g)=1+\left(\sigma_{11}(g)+\bar{\sigma}_{11}(g)\right) / 2$ for each $g \in H$. For each $\rho \in R(H), \int_{H} \rho \psi d \mu$ means the matrix $\left[\int_{H} \rho_{i j} \psi d \mu\right]$ where $\rho=\left[\rho_{i j}\right]$ and $\mu$ is normalized Haar measure on $H$.

We now construct the desired sequence $\left\{x_{v}\right\}$ in blocks with the nth block having $T_{n}$ terms. This sequence will have the property that for each complex valued continuous function $f$ on $H,\left\langle f,\left\{x_{v}\right\}\right\rangle=\int_{H} f \psi d \mu$. For a fixed integer $n$, let $p_{v n}$ denote the integral part of $n \psi\left(y_{v}\right)$. Then $\left|\psi\left(y_{v}\right) / n-p_{v n} / n^{2}\right|<1 / n^{2}$. Therefore,

$$
\sum_{v=1}^{n}\left|\frac{\psi\left(y_{v}\right)}{n}-\frac{p_{v n}}{n^{2}}\right|<1 / n .
$$

The $n$th block of the sequence $\left\{x_{v}\right\}$ will be $y_{1}, y_{1}, \cdots, y_{1}, y_{2}, y_{2}, \cdots, y_{2}$, $\cdots, y_{n}, y_{n}, \cdots, y_{n}$ where $y_{j}$ occurs $p_{j n}$ times as $j$ runs from 1 to $n$. This block has $T_{n}$ terms where $T_{n}=\sum_{v=1}^{n} p_{v n}$. The sequence $\left\{x_{v}\right\}$ has the property that $\lim _{n \rightarrow+\infty} T T_{n} / n^{2}=1$. It follows that for each continuous complex valued function $f$ on $H$, the limit of averages of $\left\{f\left(x_{v}\right)\right\}$ over the blocks in the sequence $\left\{x_{v}\right\}$ is $\int_{H} f \psi d \mu$. The limit of averages of $\left\{f\left(x_{v}\right)\right\}$ which terminate at the end of the blocks of $\left\{x_{v}\right\}$ is also $\int_{H} f \psi d \mu$. Since $\lim _{n \rightarrow+\infty} T_{n+1} /\left(\sum_{m=1}^{n} T_{m}\right)=0$, the averages of $\left\{f\left(x_{v}\right)\right\}$ which terminate in the middle of a block behave in the limit in the same way as averages which terminate at the end of blocks.

From the orthogonality of the m.a.i. functions from irreducible representations on a compact group, it is clear that $\int_{H} \rho \psi d \mu=0$ for all nontrivial $\rho$ in $R(H)$ except possibly $\sigma$ and $\bar{\sigma}$. Let us assume that $\int_{H} \sigma \psi d \mu=0$. If $\bar{\sigma}$ is a nontrivial member of $R(H)$ distinct from $\sigma$, then $\int_{H} \bar{\sigma} \psi d \mu=0$. Therefore $\psi$ is orthogonal to all $\rho \in R(H)$ except 1 . The function $1-\psi$ is orthogonal to all representations in $R(H)$. Thus $1-\psi=0$ and $\psi=1$. This is not possible since $\psi(e)=2$. Therefore $\int_{H} \sigma \psi d \mu \neq 0$.

Remark. Compare Lemma (3.2) with its abelian analogue [2, Lemma 3.1, p. 444].

We now prove the existence and uniqueness theorem concerning $D$-compactifications of $K$-separable locally compact groups.

(3.3) Theorem. Let $G$ be a locally compact group which admits a u.d. sequence. Suppose that $P$, the set of periodic representations, is an AC subset of $R(G)$. Then the sequence $\left\{x_{v}\right\}$ in $G$ is $u_{. d}$. if and only if $\left\{\phi\left(x_{v}\right)\right\}$ the image of $\left\{x_{v}\right\}$ under the natural bomomorphism from $G$ into $G_{P}$ is $u_{0} d_{\text {. in }} G_{P}$. That is, $G_{P}$ is a D-compactification of $G$ if and only if $P$ is an $A C$ subset of $R(G)$. In addition, $G_{P}$ is the only compactification of $G$ which is a D-compactification. If $P$ is not an $A C$ subset of $R(G)$, there is no D-compactification. 
Proof. Assume that $P$ is an AC subset of $R(G)$. By Lemma $(2.6) R\left(G_{P}\right)=P$. If we use the Weyl criterion for locally compact groups $\left[1, \S_{1}\right]$ we see that $\left\{x_{v}\right\}$ is u.d. in $G$ if and only if $\left\{\phi\left(x_{v}\right)\right\}$ is u.d. in $G_{P}$.

Suppose that $H$ is another compactification of $G$. We may identify $R(H)$ with that subset $S \subset R(G)$ which is an $A C$ subset of $R(G)$ and which has the property that the maximal ideal space of $B A(S)$ is $H$. The set $S$ does not equal $P$ since equality would imply that $H$ was topologically isomorphic to $G_{P}$. Let us assume first that there exists $\sigma \in S-P$. Let $Q=G_{P \cup\{\sigma\}}$, that is, $Q$ is the compactification of $G$ with respect to $P \cup\{\sigma\}$. By assumption $G$ possesses a u.d. sequence $\left\{x_{v}\right\}$. By the Weyl criterion, $\left\langle\rho,\left\{x_{v}\right\}\right\rangle=0$ for all nontrivial $\rho \in P$. Let us consider $R(Q)$ as a subset of $R(G)$. By Lemma (3.1) there is a sequence $\left\{b_{v}\right\}$ in $G$ such that $\left\langle g,\left\{b_{v}\right\}\right\rangle=0$ for each nontrivial m.a.i. function $g$ from a representation in $R(Q)$. Hence $\left\langle\rho,\left\{b_{v}\right\}\right\rangle=0$ for all nontrivial $\rho \in R(Q)$. Let $\phi_{Q}$ be the natural homomorphism sending $G$ into $Q$. For all nontrivial $\rho \in R(Q)$ we have $\left\langle\rho,\left\{b_{v}\right\}\right\rangle=$ $\left\langle\rho,\left\{\phi_{Q}\left(b_{\nu}\right)\right\}\right\rangle=0$. By the Weyl criterion for compact groups we conclude that $\left\{\phi_{Q}\left(b_{v}\right)\right\}$ is u.d. in $Q$. By Lemma (3.2) we may rearrange $\left\{\phi_{Q}\left(b_{v}\right)\right\}$ with repetitions in a new sequence $\left\{\phi_{Q}\left(k_{v}\right)\right\}$ so that $\left\langle\rho,\left\{\phi_{Q}\left(k_{v}\right)\right\}\right\rangle=0$ for all nontrivial $\rho \in R(Q)$ except $\sigma$ and $\bar{\sigma}$ but $\left\langle\sigma,\left\{\phi_{Q}\left(k_{v}\right)\right\}\right\rangle \neq 0$. Since $\left\langle\rho,\left\{\phi_{Q}\left(k_{v}\right)\right\}\right\rangle=\left\langle\rho,\left\{k_{v}\right\}\right\rangle$ for each nontrivial $\rho \in P$ we see that $\left\{k_{v}\right\}$ is u.d. in $G$. Let $\phi_{H}$ be the natural homomorphism sending $G$ into $H$. Since $\left\langle\sigma,\left\{k_{v}\right\}\right\rangle=\left\langle\sigma,\left\{\phi_{H}\left(k_{v}\right)\right\}\right\rangle=\left\langle\sigma,\left\{\phi_{Q}\left(k_{v}\right)\right\}\right\rangle,\left\{\phi_{H}\left(k_{v}\right)\right\}$ is not u.d. in $H$. Therefore $S-P=\varnothing$.

Suppose next that there is a $\sigma \in P-R(H)$. We recall that $\left\{\phi\left(x_{\nu}\right)\right\}$ is u.d. in $G_{P}$. We use Lemma (3.2) on $\left\{\phi\left(x_{v}\right)\right\}$ to obtain a new sequence $\left\{\phi\left(k_{v}\right)\right\}$ which has the property that $\left\langle\rho,\left\{\phi\left(k_{\nu}\right)\right\}\right\rangle=0$ for all nontrivial $\rho \in P$ except $\sigma$ and $\bar{\sigma}$ and $\left\langle\sigma,\left\{\phi\left(k_{v}\right)\right\}\right\rangle \neq 0$. The sequence $\left\{k_{v}\right\}$ is not u.d. in $G$ since $\left\langle\sigma,\left\{k_{v}\right\}\right\rangle=\left\langle\sigma,\left\{\phi\left(k_{v}\right)\right\}\right\rangle \neq$ 0. If $\bar{\sigma} \in R(H)$, then since $R(H)$ is an AC subset of $R(G), \sigma \in R(H)$. This is not possible. Therefore $R(H)$ contains neither $\sigma$ nor $\bar{\sigma}$. It follows that $\left\langle\rho,\left\{\phi_{H}\left(k_{v}\right)\right\}\right\rangle=$ $\left\langle\rho,\left\{\phi\left(k_{v}\right)\right\}\right\rangle=0$ for all nontrivial $\rho \in R(H)$. Therefore $\left\{\phi_{H}\left(k_{\nu}\right)\right\}$ is u.d. in $H$. Thus, if $H$ is a $D$-compactification of $G, R(H)$ coincides with $P$.

Suppose that $P$ is not an $A C$ subset of $R(G)$. Let $H$ be any compactification of $G$. We consider $R(H)$ as a subset of $R(G)$. By Lemma (2.6), $R(H)$ is an AC subset of $R(G)$. Thus the set of irreducible unitary representations of any compactification of $G$ is not equal to $P$.

Let $H$ be a $D$-compactification of $G$ and suppose $\sigma \in R(H)-P$. Let $\left\{x_{v}\right\}$ be u.d. in $G$ and let $Q=G_{P \cup}\{\sigma\}$. By Lemma (3.1) there exists a sequence $\left\{b_{v}\right\}$ in $G$ such that $\left\langle\rho,\left\{\phi_{Q}\left(b_{v}\right)\right\}\right\rangle=0$ for all nontrivial $\rho \in R(Q)$. By the Weyl criterion for compact groups, $\left\{\phi_{Q}\left(b_{v}\right)\right\}$ is u.d. in $Q$. By Lemma (3.2) we may rearrange $\left\{\phi_{Q}\left(b_{\nu}\right)\right\}$ with repetitions in a new sequence $\left\{\phi_{Q}\left(k_{v}\right)\right\}$ so that $\left\langle\rho,\left\{\phi_{Q}\left(k_{v}\right)\right\}\right\rangle=0$ for all nontrivial $\rho \in R(Q)$ except $\sigma$ and $\bar{\sigma}$ and $\left\langle\sigma,\left\{\phi_{Q}\left(k_{\nu}\right)\right\}\right\rangle \neq 0$. It follows that $\left\langle\rho,\left\{k_{\nu}\right\}\right\rangle=$ 
$\left\langle\rho,\left\{\phi_{Q}\left(k_{v}\right)\right\}\right\rangle=0$ for all nontrivial $\rho \in P$. Thus $\left\{k_{\nu}\right\}$ is u.d. in $G$, but since $\left\langle\sigma,\left\{\phi_{H}\left(k_{v}\right)\right\}\right\rangle=\left\langle\sigma,\left\{\phi_{Q}\left(k_{v}\right)\right\}\right\rangle \neq 0,\left\{\phi_{H}\left(k_{v}\right)\right\}$ is not u.d. in $H$. Therefore there is no $\sigma$ in $R(H)-P$. Let us assume next that $\sigma \in P-R(H)$. Let $Q=G_{P \cup\{\sigma\}}$. We use

Lemma (3.2) on $\left\{\phi\left(x_{v}\right)\right\}$ to obtain a new sequence $\left\{\phi\left(k_{v}\right)\right\}$ which has the property that it is u.d. in $H$ but $\left\{k_{v}\right\}$ is not u.d. in $G$. Therefore there is no $D$-compactification if $P$ is not an $A C$ subset of $R(G)$.

Remark. Let us suppose that $G$ is abelian in the previous theorem. An $A C$ subset of $R(G)\left(R(G)\right.$ is now the character group $G^{\wedge}$ of $\left.G\right)$ is simply a subgroup of $G^{\wedge}$. The set $P$ is the subset $G^{\wedge P}$ of periodic characters of $G^{\wedge}$, see $[2$, p. 436]. In this setting, the statement and proof of Theorem 3.3 almost coincide with [2, Theorem 4, p. 445].

\section{BIBLIOGRAPHY}

1. L. Benzinger, Uniformly distributed sequences in locally compact groups. I, Trans. Amer. Math. Soc. 188 (1974), 149-165.

2. I. D. Berg, M. Rajagopalan and L. A. Rubel, Uniform distribution on locally compact Abelian groups, Trans. Amer. Math. Soc. 133 (1968), 436-446. MR 37 \#3279.

3. E. Hewitt and K. Ross, Abstract harmonic analysis. Vol. I: Structure of topological groups. Integration theory, group representations, Die Grundlehren der math. Wissenschaften, Band 115, Academic Press, New York; Springer-Verlag, Berlin, 1963. MR 28 \#158.

4. - Abstract harmonic analysis. Vol. II: Structure and analysis for compact groups analysis on locally compact Abelian groups, Die Grundlehren der math. Wissenschaften, Band 152, Springer-Verlag, Berlin and New York, 1970. MR 41 \#7378.

5. L. H. Loomis, An introduction to abstract harmonic analysis, Van Nostrand, Princeton, N. J., 1953. MR 14, 883.

6. M. A. Naìmark, Normed rings, 2nd rev. ed,, "Nauka", Moscow, 1968; English transl., Wolters-Noordhoff, 1970.

805 CENTENNIAL DRIVE, CHAMPAIGN, ILLINOIS 61820 